COMMISSION No. 19

\title{
ROTATION OF THE EARTH (ROTATION DE LA TERRE)
}

Report of meetings: 3-10 August 1988

president: W.J. Klepczynski

3 August :

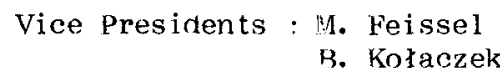

Joint Comnission Meeting 1 , JCMl-1

The JCM-1 was a meeting on the topic "For milliarcsecond or better accuracy", organized by $P$. Seidelman, jointly for comnissions $4,8,19,24,31,40$. The meet ing included 11 invited papers concerning observational accuracies, theoretical developments and computational considerations, and the reports of four working groups, on The use of millisecond pulsars, Nutation, Astronanical constants, and Reference Systems. The report of JCM-1 will appear in Highlights of Astronomy.

4 August, $9: 00$

Chair : W.J. Klepczynski
Commission 19 - Business meeting I

Secretary : A. Babcock

Announcements

- The IAU Executive Committee appointed 1)r. Yatskiv as the IAU representative to the IERS bi recting Boam.

- The Comnission 19 Organizing Committee will meet (on Friday, 5 th) to discuss: nominations to the Organizing Comnittee, proposed new members (5 proposed so far), and consultants

Rermarks by the President : W. Klepczynski reviewed the accomplishments on the last three years, especially (1) the establishment of the IERS and (2) the studies of atmospheric driven irregularities in the Earth rotation. He observed that much time has been spent during Commission 19 meetings on reports from individual members on their own work, but reminded that the function of the IAU is to coordinate joint efforts between astronomers at different institutions or between scientists in different disciplines (i.e. IERS, MERI'T).

Election of officers : The folloging nominations had been made:

President: Martine Feisse1, Vice President: Ye Shu-hua. Concern was expressed by $B$. Kołaczek that M. Feissel should not head both IERS Central Bureau and Comnission 19 since it was established tradition that those actively involved in an IAU service would not be eligible to hold and office in which they would make policy recomnendations regarding the service. W. Klepczynski responded that (1) IERS responsability is shared between the Central Bureau and the Technique Coordinators, so that neither of $f$ icer has full control and (2) suitable candidates for Commission office are very scarce and should not be disqualified simply because they are active observers or analysts. This opinion was strongly endorsed by 1 . Wueller. The vote was taken and the nominees elected with 16 for, 3 abstaining. 
Review of resolution on Reference Systems The report of the WG on Reference Systems had been presented at the JCM-1 by its president J.A. Hughes. A resolution had been prepared, which recommended the continuation of the WG, and the formation of Study Groups under it, on special ized topics. The discussion on this resolution was open. M. Feissel remarked that D. McCarthy had suggested a study group (SG) be formed on Links between Reference Frames. D. McCarthy said this would avoid having SG's on individual reference frames having to cover too much territory. B. Gilinot considered that this was really the job of IERS, but it was explained that IERS only concerns itself with the reference frames of the techniques in its solution, whereas the optical reference frame is also of interest. Ye suggested the creation of a new comnission on reference systems to replace Commission 31. This suggestion had been made at New Delhi. 1). M.CCarthy recalled, but the IAU had not responded to the recommendation. After some discussions on the various possibilities of new organization, it was decided to add an SG on Reference Frames to the 1 ist of SG'S in the resolution of JCM-1. It was also recomnended that SG's on Time, Nutation, Origins, and Astronomical constants be included in the Working Group on Reference Sys tems.

Recommendation on IAU contribution to FAGS There was a brief discussion of the recommendation that the IAIJ increase their support to the Federation of Astronomical and Geophysical Data Analysis Services (FAGS). This recommendation requests that the Presidents of Commissions 19 and 31 coordinate an action with the Presidents of IAU cammissions concerned with the FAGS Services.

New astrononical organization in Japan : There was a discussion of the new National Astronomical Observatory in Japan, which encampasses the functions of ILOM as well as many other astronomical institutions.

History of IPUS and BIH : D. McCarthy suggested that now that both the IPUS and $\mathrm{BIH}$ have been discontinued, their histories should be written as soon as possible. It was suggested that a symposium be held on the history of these organizations, but this was not strongly endorsed. I. Mueller suggested a formal resolution to direct the heads of the BIH and IAWS to write the history of their organizations. B. Wackernagel pointed out that Commission 41 (History of Astronomy) should be involved in this effort. Ya. Yatskiv said that a session on the history of the ILS, IPMS, and BIH could be included in a meeting scheduled for next year in Leningrad. I. Mueller thought a session at the next IAU General Assembly would be preferable. In any case, the resolition should be written, and addressed to $B$. Guinot and $\mathrm{s}$. Yumi .

4 August, $11: 00$

Chair : W.J. Klepczynski

\section{Commission 19 - Reports of Services}

\section{Secretary : J. Popelar}

Bureau International de l'Heure (BIH)

M. Feissel presented the report on the earth rotation activities of the Bureau

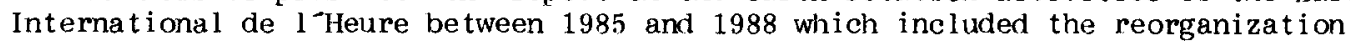
into the new International Earth Rotation Service on January 1, 1988. She reviewed the activity highlights as follows.

- Farth Rotation Parameter (ERP) solutions computed on a weekly, monthly and annual basis have incorporated all high precision results provided by VLBI, lunar and satellite laser ranging (LLR, SLR) measurements.

- A homogeneous combined ERP series have been compiled for the period 1962-1987 at 5-day intervals; its precision reached 0.002" for polar motion and 0.2ms for UC1 during 1984-1987, which represents an order of magnitude improvement for the annual and shorter term variations and a fivefold improvement for longer term changes over the 25 years. 
- An ERP solution for optical astrometry based on the IAU 1980 theory of nutation has been obtained for all data since 1978: A deterioration of long tem stability has been detected after 1984 which is reflected in annual tem fluctuations and a drift in they coordinate of the pole.

- BIH Terrestrial System (BIS) has been re-defined in 1984 and is now based on a set of the cartesian coordinates of 64 sites where at least two of 13 avai lable space geodesy networks overlap; the French Institut Geographique National (IGN) carried out the global adjus trent which also produced the transformation parameters between the input networks and BTS; the BIS (1987) makes use of the Minster and Jordan plate motion model AVD-2.

- An extragalactic Celestial Reference Frame consistent wi th the BIS(1987) has been derived using four radio source catalogs; the coordinates of 23 prinary sources and 205 additional sources, as well as the relative orientation between the original frames and the combined frame have been de ternined.

- Series of pseudo Universal time based on Atmospheric Angular Monentum (AAM) have been established to facilitate studies of the seasonal and higher frequency variations in the length of day and for possible use in the quick look ERP solutions in future.

- The last BIH Annual Report for 1987 has been produced and distributed as have been hard copies of the Circulars A to F; at the same time greater use of the computer networks (GE Mark III, EARN/BITNEF and SPAN) has been encouraged for data dissemination.

- In 1988 the Earth rotation section of the BIH, associated with groups in IGN and Bureau des Longitudes has been transformed in to the Central Bureau of IERS, continuing to maintain the data bases and assuring system cont inui ty.

In the discussion following the BII report the commission president and $\mathrm{G}$. Winkler expressed concern regarding the deterioration of the optical astronetry results in recent years which would jeopardize any comparison between the classical and the new space techniques. In her reply Dr. Feissel attributed this fact mainly to reduced number of observations as many observatories have been phasing out their optical astrometry programs. Prof. Ye pointed out that more than 50 instruments are still in operation and the Shanghai Osservatory has assumed the responsibility of the global analysis center which will continue to facilitate comparisons wi th the optical technique in the future.

\section{International Polar Motion Service (IPMS)}

Dr. Yokoyama presented the activity report of the International Polar Motion Service for 1985-1988. He reviewed the organization of the new National

Astronomical Observatory (NAO) of Japan which was established on July 1, 1988 and incorporates among others the forner International Latitude Ooservatory of Mizlusawa, the host organization of the IPUS between 1962 and 1987. The Earth Rotation Division and the Mizusawa Astrogeodynamics Ooservatory (NACM) will continue the activities related to the Earth rotation, geodynamics and gravity.

During the last period the IPPS continued as the operational and data analysis center for the optical astrometry as part of the extended MERI' $C$ campaign. An extensive computer data base has been compiled and includes :

- all ILS observational records and results between 1899 and 1979 ,

- all IPMS optical astronetry data, ERP results and related information between 1962 and 1987, 
- complete set of computer programs for the reduction and analysis of optical astrometry observations, star catalog adjustment and global FRP estimation including evaluation of individual station biases.

The data base is available on request and it is ready for re-processing after the Hipparcos star catalog becomes available.

Since $1985 \mathrm{~K}$. Yokoyama and the IPMS staff have been preparing for the transition to the newly organized IERS. The NAOM has taken the initiative and participates in the IERs activities by organizing :

- the VLBI center for IRIS-P network while actively promoting the VEIZA Project, the Japanese VLBI for Earth Rotation and Astrometry; the IRIS-P network has carried out monthly experiments using Kashima, Fairbanks, Ft. Davis and Richmond stations since April 1987 .

- the VLBI data analysis center which developed data reduction and ERP estimation software; IRIS-P and IRIS-A experiments have been processed since the beginning of 1987 and detailed comparison of the results with NGS has been carried out,

- the computing center for evaluating atmospheric excitation functions using the Japanese Meteorological Agency data.

The NAOM also carries out research into earth tides, ocean loading and other geodynamic effects for which it operates a transportable absolute gravimeter, a superconducting gravimeter and tiltmeters.

During the discussion Dr. Djurovic asked if effects of ground water level changes are taken into account in data reduction. Dr. Yatskiv pointed out that these effects at the Poltava Observatory have been smaller than 0.001 " which is be low the accuracy of current measurements. D. McCarthy noted that information on ground water levels is difficult to obtain and some countries would not release it even if available. M. Gaposhkin inquired about use of laser ranging techniques in Japan. K. Yokoyama responded that there are no plans of NAO to use LILR or SLR for earth rotation studies but he referred to the Japanese Hydrographic Institute which has investigated use of SLR.

4 August, 14:00 Comnission 19-Time series analysis

Chair : D.D. McCarthy

The following contributions were presented

- Time series analysis as a tool for accurate access to reference systems (M. Feissel and D. Gambis).

- Time series analysis and studies of Earth rotation (Ye. Shu-hua).

- Earth rotation studies at Jet Propulsion Laboratory (J.O. Dickey).

- A note on the usage of maximm entropy spectral analysis in the determination of Earth Rotation Paraneters (R. Vicente and H. Lenhard $t$ )

- Time series analyses of Earth rotation at Kiev Ooservatory (Ya. Yatskiv).

- Non-linear spectra and the Earth's rotation (T.H. Eubanks).

- Analysis of the zonal tides of UT1 from 1962 to 1988 (J. Hefty and N. Capi taine).

- On the solar origin of the fifty-day fluctuation of the Earth's rotation and a trnospheric circulation (Djurovic and P. Pâquet).

- Time series analyses of Lunar Laser Ranging (Ch. Veillet). 
Ten contributions for the sessions devoted to "Reference Frames" pernitted to overview the major topics, from the concepts to the realisations of the various systems.

The basic concepts on the definitions and realisations of the reference systems have been described in details by I. Mueller. SLR, LLR or VLBI ground-based observatories are the basis of a conventional terrestrial system (CTS) realised through a combination of sites and techniques. This combination has to take into account all the infornation available (colocation, local survey, ...) and to include all the unknowns relater to the motion of the sites, scale factors for ERP series, etc.

The intercomparison of the various celestial reference frames, as pointed out by Mrs Ye, has to be carefuly made. The differences in formulation and definition can be clear, but their implementation in the various techniques as well as in the data analysis is not straightforward. For the next decades, a four-dimensional reference system is needed and could be the goal of a new commission.

Links between VLBI and dynanical reference frames made at JPL have been described by J. Dickey. Differential VLBI observations of quasars and Mars orbiters are a possible technique for such a link, as well as VLA observations of Galilean satellites, Titan. Uranus and Neptune. None of these observations were included in DE125, but they would improve the ephemeris. VLBI and optical frame agree at the $0.02 "$ level for the northern hemisphere, at the $0.1 "$ level for the southern. This link should improve in a near future through VLBI observations of millisecond pulsars, differential VLBI observations of Phobos and Calileo, VLA observations of outer planets, and wi th the use of Hipparcos and space astrometry.

Four extragalactic radio sources catalogues (GSFC, JPL, NGS and extended GSFC) have been compared and combined after a selection of primary sources in a work made at the IERS Central Bureau presented by F. Arias. The various origins are in a 0.002 " radius circle, but there are systematic deformations between IPL and NGS or GSFC catalogs, as a function of source declination. The resulting catalog has a total of 228 sources, including 23 primary ones. It has only 8 primary sources in the southern hemisphere, but should be extended in a near future.

On the Earth, the IRIS-A and IRIS-P networks are both operational. K. Yokoyaina presented briefly IRIS-P. the discrepancies between the procedures used in these networks have been minimized.

Using the VLBI observations, T. Herring presented his last results on nutation corrections to the IAU (1980) nutation series. It is very clear now that the observed semi-annual and annual terms are different from the conventional values, as well as the 13.7 days term. V. Dehant presented some possible explanations of these discrepancies. She showed how the input of the Earth interior model can be changed in an attempt to explain this disagreement. In fact, an improved external tidal potential doesn' $t$ explain it. Taking into account the mantle anelasticity gives still larger differences. Variations of the core flattening added to the previous modifications permit to obtain a better agreement between the theory and the observations, but are not sufficient. Variations of the pressure at the core-mantle boundary have to be included in a near future. 
Recent results on precession corrections from LLR have been presented by $\mathrm{J}$. Dickey. The uncertainty is still large, but should rapidly decrease as the modern accurate data will have a higher weight.

On a more general basis, D. McCarthy discussed the use of standards for reference systems. As they are both a basis for the comparisons and a tool for the observations. there is a natural conflict on their nature. Errors in standards are in fact compensated by errors in Earth Orientation Parameters (BOP), and very often FuP become a standard for other users. Finally, the analysis leads to improve the standards continuously. If we use different standards, do we understand really the influence of these differences? Many points and questions which make the standards an open problem.

Ya. Yatskiv reported on the effect of the pole tide in the various techniques contributing to BOP determination, and proposed to add these effects to the standards.

8 August, 14:00 Comissions 19 and 31 - International Earth Rotation Servic

Chair : Ya. Yatskiv (session 1) Secretary : G.A. Wilkins
G.A. Wilkins (session 2)
I.I. liveller (session 3)

The first two sessions of the joint meeting of Commissions 19 and 31 on the International Earth Rotation Service (IERS) were devoted to the presentation of papers on various aspects of the development and operation of IERS, which replaced the Earth-Rotation Section of the Bureau International de 1 Heure (BIH) and the International Polar Motion Service (IPUS) on 1988 .January 1. The afternoon session was mainly devoted to an open discussion on matters relating to the current and future operation of IERS. In addition, W. Markowitz presented a short paper on the secular motion of the pole.

History. P. Pâquet reviewed the development of the international services for time and polar motion. The International Latitude Service (ILS) was founded in 1891 on the basis of clear evidence of variations of astrononical latitude, but regular observations from 5 stations at a common latitude did not start until 1899 . The introduction of wireless (radio) tirne signals in 1910 led eventually to the setting up in 1919 of BIH in association with the Time Commission of the newly founded IAU. The improved precision in the detemination of time led in 1955 to the establishment by $B I H$ of a rapid service for latitudes so that corrections for polar motion could be applied in the deternination of UT (and hence in the deternination of longitude). The ILS was replaced by IPMS in 1962 . The introduction of atomic time and the use of space geodesy data to determine the Earth rotation parameters led to considerable changes in the work of BIH.

G. A. Wilkins reviewed very briefly the prograrme of Project MEKI'C (to Monitor Earth Rotation and Intercompare the Techniques of observation and analysis) and the associated COlEs programe for the establishment of a new Conventional Terrestrial System for defining positions accurately on the Earth's surface. The results had shown that the techniques of VLBI and of laser ranging provided precise and complementary ways of monitoring the changes in the rotation-vector of the Earth and of establishing celestial and terrestrial reference frames. These are now the primary techniques used in IERS, but the Service will take into account all avai lable data of high precision.

VLBI. 1). S. Robertson reviewed the status of the VLBI operations that contribute to IERS. There are now $12 \mathrm{stations}$ in regular operation in the northern 
hemisphere, but only one in the southern hemisphere. Other stations contribute occasionally and more are being developed or planned. The radio-source catalogue that defines the celestial reference frame is 1 imited to 23 prinary sources between latitudes $80 \mathrm{~N}$ and $50 \mathrm{~s}$. Many very small effects must be modelled in order to natch the precision ( $\left.0.0131^{\prime \prime}\right)$ of the observations; corrections to the standard values of nutation in longitude and obliquity are detemined from the observations. There is good agreement with the SLR results for the coordinates $x, y$ of the pole. Daily deteminations of UT are made using transatlantic baselines; the results confirn Yoder's earth-tide norlel wi th errors of order of $0.1 \mathrm{~ms}$. T. A. Herring gave further details of the deteminations from the VLBI data of the corrections to the IAU (1980) nutation series. Terns with periods less than one year are deternined well, but as yet it is not possible to separate fully the effects of precession from those of the long-period terms.

Laser Ranging. B. E. Schutz presented a sumnary of his report as Coordinator for satellite laser ranging; this technique contributes to the IERS determination of $x, y$, UT and length of day $(\omega)$, and also to the determinations of the temporal variations of the gravity field and tectonic motions of the stations; moreover, the station coordinates are determined in a frame whose origin is at the centre of mass of the Earth. About 20 stations contribute regularly to IERs; it would be useful to have more stations in the centre of the Eurasian plate and in the southern hernisphere. The operational centres at Aust in (CSR) and Delft (IUT) make independent solutions using 3 -day and 5-day arcs, respectively. The results differ by about $0.001 "$ in $\mathrm{x}, \mathrm{y}$ and 0.2 ins in UT; similar differences are found between SLR and VLBI results. The launching of more satellites with retroreflectors may reduce the coverage of LALiEXs and so require a new approach to the determination of the earth-orientation parameters; the precision of measurement should be reduced to about $1 \mathrm{~mm}$, but it may not be possible to model the atmospheric transmissions to this accuracy.

C. Veillet reported that there are only three lunar laser ranging stations in regular operation at present, but several others should be provided in a year or two. The accuracy of ineasurement of the range is about $3 \mathrm{~cm}$. Estimates of UT can, however, be determined from observations at one station with very little computation. The values of UT are model dependent and the choice of lunar ephemeris has a significant effect on the scatter of the results. The LLR and VuBI results differ at the level of about $0.05 \mathrm{~ms}$ after fitting. The values of the nutation corrections detemnined from the LlR data are about $50 \%$ larger than those from VLRI, but the estimates of the correction to the precession constant are similar. The analyses continue to confinn Einstein's General Theory of Relativity. Better results are to be expected when the new stations also contribute data regularly.

Standards. D.I. McCarthy, the chaiman of the IERs Working Group on standards, said that IERS is now fomally operating under the MERIT standards, but it is clear that some changes are needer. Unfortunately, the MERIT standards are not always being used, and some of the members of the group have been very slow in producing revised drafts of the chapters for which they have responsibility. He questioned the value of standards in such circumstances. G.A. Wilkins and I.I. Mueller both stressed the value of having clearly specified up-to-date standards to simplify the combination of results obtained by different techniques of observation and the comparison of results obtained using different models.

Central Bureau. M. Feissel described the work of the Central Bureau of IERS. It is based at the Paris (bservatory, but has the support of the Institut Geographique National and the Bureau des Longitudes in respect of the work on terrestrial and celestial reference frames. Two Sub-Bureaux contribute to the general services of IERS (see following reports). In addition IERS depends on 
the work of 3 coordinating centres (in 2 countries), 20 analysis centres (in 10 countries) and 50 observing stations (in 30 countries). She also stressed that standardisation is necessary if the data and results from these many sources are to be combined to give useful results. The initial realizations of the IERS reference frames will be based on the BIH combined solution for 1987; there are 64 primary stations in the terrestrial frame and 23 primary sources in the celestial frarne; more primary points are required, and secondary points are required to make these franes more readily accessible. The comparisons of the individual solutions for the earth-orientation parameters show that the errors are of the order of 0.002 " ; the cambined solution is published at an interval of 0.05 years, but values are also available at intervals of 1,3 and 5 days. Several series of circulars are issued (weekly, monthly, and as appropriate) and the Annual Report will give the final results from the Coordinating Centres and the combined solution; Technical Reports will give more infornation about the methods that are used; the first will contain the new IERS Standards. The IERS results are also available on-line.

D.D. McCarthy described the activities of the Rapid Service Sub-Bureau, which is operated jointly by the U.S. Naval Observatory and the National Geodetic Survey in washington, D.C. It issues the Weekly Bullet in A, which contains estimates of the earth-rotation parameters that are based on quick-look data received up to two days before publication and on prediction models. The differences between the quick-look estimates and the values published later in the Monthly Bulletin B, issued by the Central Bureau, are gradual ly being reduced and are now about $0.002-3^{\prime \prime}$ in $\mathrm{x}, \mathrm{y}$ and $0.3 \mathrm{~ms}$ in UT. The errors in the predictions are about $0.015^{\prime \prime}$ in $x, y$ and $30 \mathrm{~ms}$ in UT after 80 days. The relative contributions of the different techniques differ between polar notion and UT, and also differ fran those for Bulletin $\mathrm{B}$. At present the forecasts of the angular momentum of the atmosphere are not used in the predictions of the earth-rotation paraneters. In the luter discussions, J. O. Dickey and $M$. Feissel said that the use of forecast values of AAM does improve the predictions of UT.

J. O. Dickey apologised for the absence of ..A. Miller of the (U.S.) National . Neteorological Centre, which has agreed to operate a Sub-Bureau for Atmospheric Angular Monentun (AAM) for two years from the spring of 1989 . (At present, the U.K. Meteorological of $\mathrm{fice}$ and the European Centre for Hedium-Range Weather Forecasting send their data directly to the Central Bureau.) The main purpose of the Sub-Bureau will be to consolidate the synoptic and forecast values of AAM received from the different agencies, including those of the Japanese Meteorological Agency. The close correlation between the variation in LOD and AAM is of great scientific interest and it is hoped that forecasts of AAll will prove useful in the prediction of UT.

General discussion. The Chaiman of the third session, I.I. Mueller, suggested that the first topic of discussion should be the use of standards in IERS. He pointed out that in the MERIT project it had been agreed that all analysis centres should provide results based on the MERIT standards, but they were invited to also make analyses using other constants or models and to explain the differences in the results. W.E. Carter considered that it is sufficient if the analys is groups specify how the constants and models used to obtain their results differed from those in the standards; he and T.A. Herring considered that the errors introduced by using poor standards are unacceptable. This led to a consideration of the requirements of different types of users of the IERS services. In general, the operational community does not need results of the highest-possible precision, but it does require reliable, consistent stable series based on well-documented published standards and procedures. On the other hand, the research community is striving to obtain results of the highest-possible accuracy so that it can investigate the physical causes and implications of the variations in the earth-rotation vector; to this end it wishes to use the best available constants and models, but it must also be prepared to reprocess old observational data, or, in appropriate circunstances, to apply differential corrections to old results. 
T. A. Herring pointed out that the changes would not be noticed by the operational comminity. B. Guinot stressed that in order to make possible the combination of results from different techniques it is essential that certain standards be used in comon. The general conclusion of the discussion appeared to be that new IERS Standards are required as soon as possible, and that the birecting Board should take into account the wide range of views expressed during the discussion.

W. Warkowitz presented an analysis of the motion of the mean pole as obtained from independent measurements based on optical astronetry, VLBI and SLR. He showed that the secular motion of the pole over 1976-1987 derived from the different series are in agreement, which confims the reality of this motion.

In the remaining part of the session, J.o. Dickey drew attention to the desirability of obtaining earth-rotation paraneters at an interval of less than one day for comparison with the variations in AAM ; she suggested that a coordinated campaign of observations should be organized during the TDPEX mission. C. Veillet consideren that LLR quick-look estimates could be useful for the rapid service, especially when more stations are in operation to reduce the gaps in the data due to poor wea ther.

8 August, $16: 00$

Commission 19 - Report of the Wi on Optical Astrometry

Chair : Ye Shu-hua

Secretary : B. Kołaczek

At this session the report of the Working Group on classical astrometric observations were presented by its chairperson, Ye Shu-hua. She reviewed the activity of the Working Group which consisted of meetings and Circular letters in which the future role of the classical astrometric observations in determinations of the ERP as well as in variations of latitude and Uro was discussed.

The following conclusions were presented :

- ERP determination based on the classical astrometric observations will be carried old starting with January 1, 1988 for several years by Shanghai Observatory. At present, about 55 instruments participate in this ERP determination.

- A Working Group on re-reduction of the optical astrometric ERP series based on the HIPPAROOS system has to be establ isher.

- Monitoring of the local vertical variations in the region of seismic activity by the classical astrometric observations of time and latitude are being carried on in some observatories. The Beijing Observatory in China would like to act as the analysis center for these effects.

- Linkage of stellar reference systens connected with re-observations of star catalogues in order to compare them with the HIPPAkOOS system ought to be organized by the IAU Cormission 8 .

$J$ in Wen jing, the chief of the First Division of the Shanghai Observatory, who is responsible for the ERP determinations from classical astrometric observations presented the first report with the EKP results determined in the first three months of 1988 .

Tian Jing presented the results of the first successful prediction of the earthquake in China on the basis of the classical astrometric observations of latitude and Uro variations.

K. Yokoyama presented a proposal of a resolution for establishing a Working Group on re-reduction of classical astrometric series of the ERP. The resolution was accepted. The Comnission 19 will establish membership of this Working Group. 
The resolution reads as follows.

COMMISSION 19

\section{REOOGNIZING}

1. the importance of optical astrometry observations for Earth rotation studies accumulated since the last century;

2. the inconsistency of celestial reference frames and methods of reduction hi therto used by independent stations;

3. the significance of the fortheoming HIPPAROAs catalog in forning a consistent optical reference frame tied to a radio reference system; and

4. the necessity of recomputing optical astrometry Earth orientation parameters using a consistent method based on the HIPPAROOS catalog,

\section{REOOMLNISS}

setting up a Working Group to investigate which observatories have observations available in a suitable form for rereducing them, under the chaimanship of $J$. Vondrak, which will report to the XXIst General Assembly with reconmendations for:

a. the method of computing Earth orientation parameters, and

b. ways of implementation.

10 August, $14: 00$

Chair : W.J. Klepczynski

\author{
Comnission 19 - Business II \\ Secretary : M. Feissel
}

Ye Shu-hua had informed the commission president that, having been appointed as vice-president of the IAIJ, she wished to withdraw from the function of Vice-president of Comnission 19. B. Kolaczek was naminated by the cammission president, and elected by the nembers as the new vice-president of the cannission.

W. Klepczynski presented the list of the new Organizing Comnittee, members, consul tants and comnission mernbers.

Organizing Cormittee 1988-1991 : M. Feissel (President), B. Kolaczek, (Vicepresident),

P. Brosche, W.E. Carter, J.O. Dickey, D.M. Djurovic, Ji, Wen-jing, N. Mi ronov, D.D. McCarthy, M.G. Rochester, T. Sasao, B.E. Schutz, J. Vondrak, G.A. Wilkins.

Consultants 1988-1991 : T. Herring, H. Schuh, V.I. Sergienko, D.E. Smith.

New Menbers : E.F. Arias, A. Babcock, C. Boucher, M. Lehmann, X X Newhall.

Working Group on Reference Systems : The joint resolution of comnissions $4,7,8,19,20,24,31,33,40$ concerning the continuation of the Working Group on Reference Systems was adopted (it became later IAU resolution C2 : WGRS - A continuing intercommission project).

Proposed future meetings : A colloquium on Reference Systems will be organized in 1990 by the US Naval Observatory, to review the different topics studied by the Working Group on Reference Systems. A Symposium on Reference Systens for Astrometry and Geodesy will be held in San Juan (Argentina) in 1991, in conjunction with the 21 st IAU General Assembly. 
International Earth Rotation Service : K. Yokoyama informed the commission that he had been elected as Chaiman of the IERS Directing Board at the meeting held on August 9. The next meetings of the IERS Di recting Board are scheduled for March and August 1989. The IEls Standards should be finalized by the end of 1988 .

10 August, $16: 00$

Menbers reports

Chair : W.J. Kl epczynski

Secretary : J in Wen- $\mathrm{j}$ ing

3. Kolaczek presented a report on the prediction of short periodical variations of polar motion, based on the consideration of the main camponents in the 20-100d period range.

The topic of second presentation is the contribution of lieteorological Factors in Chinese Area to the Excitation of the Earth's Rotation reported by Sun Yong-xiang, the Institute of Geodesy and Geophysics, Academia Sinica, China. The excitation function $\psi_{1}$ and $\psi_{2}$ of air inass redistribution during 1970-1980 and axial component $\chi_{3}$ of AAll from April 1981 to December 1983 have been estinated by using the global observed meteorological data, especially including Chinese mainland data. Although the area of Chinese mainland covers only $1.9 \%$ of Earth surface area, the contributions of the Chinese area to the excitation function of air mass distribution are $14 \%$ in $\psi_{1}$ and $20 \%$ in $\psi_{2}$ and for axial component $\chi_{3}$ of AAil, the contribution of Chinese meteorological data may reach $4 \%$ of the global value. It is very inportant to utilize data of Chinese meteorological stations for calculating $\psi_{1}$ and $\psi_{2}$, and $\chi_{3}$.

Sumary of the resolutions and decisions adopted by Commission 19

Commission resolution on the reduction of past optical astrometry observations in the HIPPAROUs celestial frame, and setting up a Working Group.

IAU resolutions on

- Wits - A continuing Intercomission Project (C2)

- IAU Cont ribution to FAús (C9)

- Neerled History on BIH and IPMS (C10). 